\title{
Short and narrow flag leaf1, a GATA zinc finger domain-containing protein, regulates flag leaf size in rice (Oryza sativa)
}

\author{
Peilong He${ }^{1}$, Xiaowen Wang ${ }^{1}$, Xiaobo Zhang ${ }^{1}$, Yudong Jiang ${ }^{2}$, Weijiang Tian ${ }^{1}$, Xiaogiong Zhang ${ }^{1}$, Yangyang Li ${ }^{1}$, \\ Ying Sun ${ }^{1}$, Jia Xie ${ }^{1}$, Jile $\mathrm{Ni}^{1}$, Guanghua $\mathrm{He}^{1 *}$ and Xianchun Sang ${ }^{1 *}$
}

\begin{abstract}
Background: The flag leaf of rice (Oryza sativa L.) is an important determinant of plant type characteristics and grain yield. Identification of flag leaf mutants of rice is crucial to elucidate the molecular mechanism of flag-leaf development, and for exploitation of rice germplasm resources.

Results: In this study, we describe a mutant designated short and narrow flag leaf 1 (snfl1). Histological analysis showed that the length of epidermal cells and number of longitudinal veins were decreased in the flag leaf of the snfl1 mutant. Map-based cloning indicated that a member of the GATA family of transcription factors is a candidate gene for SNFL1. A single-nucleotide transition at the last base in the single intron of snfl1 led to variation in alternative splicing and early termination of translation. Complemented transgenic plants harbouring the candidate SNFL1 gene rescued the snfl1 mutant. Analysis of RT-PCR and the SNFL1 promoter by means of a GUS fusion expression assay showed that abundance of SNFL1 transcripts was higher in the culm, leaf sheath, and root. Expression of the SNFL1-GFP fusion protein in rice protoplasts showed that SNFL1 was localized in nucleus.

Conclusions: We conclude that SNFL1 is an important regulator of leaf development, the identification of which might have important implications for future research on GATA transcription factors.
\end{abstract}

Keywords: Rice (Oryza sativa), Alternative splicing, Short and narrow leaf, Map-based cloning, GATA zinc finger domain

\section{Background}

Rice is one of the most important cereal crops in the world and is also the staple food for nearly $50 \%$ of the world's population [1]. However, the total area of arable land is decreasing concurrent with rapid industrial development, therefore maintenance or increase in the yield of rice is strongly dependent on the improvement of grain yield per acre. Improving plant type is essential in increasing this yield. As a model monocotyledonous plant, the elucidation of physiological and biochemical mechanisms in rice are important for research on cereals and monocotyledonous species.

\footnotetext{
* Correspondence: heghswu@163.com; sangxianchun@126.com ${ }^{1}$ Key Laboratory of Application and Safety Control of Genetically Modified Crops, Rice Research Institute of Southwest University, Academy of Agricultural Sciences, Southwest University, Chongqing, China Full list of author information is available at the end of the article
}

Among the rice and other major cereals, the uppermost three leaves, especially the flag leaf, are the main source of the carbohydrates that eventually accumulate in the grains [2-5]. Previous studies of rice leaf phenotypes have focused on mapping of quantitative trait loci, and relatively few genes responsible for leaf phenotypes have ever been cloned. Rice leaf elongation is mostly determined by longitudinal cell division, cell elongation, and cell arrangement. For example, leaves of the rice dwarf mutant dwarf and gladius leaf 1 (dgl1) are shorter and the edges of the leaf tips more rounded than those of the wild type (WT). The reason of the abnormal phenotype of $d g l 1$ is that the longitudinal epidermal cells are not well elongated and the cells are distorted and bulky, leading to an abnormal cell file. [6]. An additional rice mutant with an aberrant leaf length, Oryza sativa ent-kaurene synthase 2 (osks2), produces short and dark green leaves in the seedling stage.

(c) The Author(s). 2018 Open Access This article is distributed under the terms of the Creative Commons Attribution 4.0 International License (http://creativecommons.org/licenses/by/4.0/), which permits unrestricted use, distribution, and reproduction in any medium, provided you give appropriate credit to the original author(s) and the source, provide a link to the Creative Commons license, and indicate if changes were made. The Creative Commons Public Domain Dedication waiver (http://creativecommons.org/publicdomain/zero/1.0/) applies to the data made available in this article, unless otherwise stated. 
The short leaves of osks 2 is a result of the abnormal arrangement of the longitudinal mesophyll cells of osks2 leaves, which were packed more tightly than in the WT [7]. Overexpression of Oryza sativa PATATIN-RELATED PHOSPHOLIPASE A III (OspPLAIII $\alpha$ ) results in the decreased length of rice leaves. OspPLAIII plays an important role in rice vegetative growth. Furthermore, high activity of OspPLAIII can suppress cell elongation [8]. The rice mutant brassinosteroid-dependent 1 (brd1) produces short leaves, the mesophyll cells of $b r d 1$ leaf blades are arranged more tightly than those of the WT, and intercellular spaces in the leaf blade of $b r d 1$ plants are smaller than those of the WT. In addition, motor cells and epidermal cells along the longitudinal axis of the $b r d 1$ leaf blades are much shorter than WT cells. All of these traits contribute to the short-leaf phenotype [9].

Variations in rice leaf width are mainly due to changes in vascular bundles and cell division. Many genes responsible for narrow leaf phenotypes have been cloned, such as NARROW LEAF 1 (NAL1) [10], NAL2 [11], NAL3 [11], NAL7 [12], NARROW AND ROLLED LEAF 1 (NRL1) [13], and ABNORMAL VASCULAR BUNDLES (AVB) [14]. Leaves of the nal1 mutant are narrower than those of the WT, and the cause of the abnormal phenotype was indicated to be a decrease in the number of longitudinal veins. NAL1 affects vascular patterns of rice and polar auxin transport, and plays an important role in controling lateral leaf growth [10]. The nal2/3 double mutant produces narrow-curly leaves, which result mainly from fewer longitudinal veins and reduced lateral-axis outgrowth. NAL2 and NAL3 are paralogs that encode an identical WUSCHEL-RELATED HOMEOBOX 3A (OsWOX3A; OsNS) transcriptional activator, which is involved in lateral-axis outgrowth and vascular patterning in leaves, organ development, development of tillers and lateral roots in rice, and lemma and palea morphogenesis in spikelets [11]. The $n r l 1$ mutant exhibits a reduced leaf-width phenotype. Microscopic analysis indicates that leaves of the $n r l 1$ mutant have fewer longitudinal veins compared with the WT [13]. The $a v b$ mutant causes narrow leaves. The reason of the abnormal phenotype of $a v b$ leaves is that the number of vascular bundles in the aerial organs is reduced. $A V B$ plays an important role in maintenance of the normal cell division in lateral primordia development. [14].

In the present study, we identified a short and narrow flag leaf 1 (snfl1) mutant of rice and isolated the gene responsible using a map-based cloning strategy. SNFL1 encodes a GATA zinc finger domain-containing protein. The present findings indicate the involvement of SNFL1 in the development of the epidermal cells, longitudinal veins, panicle length, number of panicles, and 1000-grain weight, demonstrating the numerous important roles of this gene in rice growth and development.

\section{Results}

Phenotypes of the snfl1 mutant

To investigate the molecular mechanisms that regulate rice leaf development, we used ethyl methanesulfonate (EMS) mutagenesis to generate a population of $\mathrm{M}_{2}$ seedlings derived from $1 \mathrm{~kg}$ of rice seeds (about 40 thousand) from the cultivar 'Jinhui10'. We screened one leaf of mutants showing an altered leaf size, which had been continuously planted for many generations, and for which the mutant traits were stable and inherited. One such mutant, snfl1, was selected for detailed study. The absence of a significant difference in plant height between WT and snfl1 (Fig. 1a; Additional file 1: Figure S1). However, the flag leaf length of snfll was dramatically decreased by about $83.28 \%$, and the flag leaf width decreased by about $67.69 \%$ compared with the WT (Fig. 1b-e). Similarly, the length of the second and third leaves of snfl 1 were decreased, whereas the width of the second leaf showed no significant difference, and the width of the third leaf was markedly increased, compared with those of the WT (Additional file 1: Figure S1). In addition, the snfl1 mutant exhibited other abnormal phenotypes, such as reduced panicle length, decreased numbers of primary and secondary panicles, reduced number of grains per main panicle, seed setting rate and 1000-grain weight, and overgrown culms (Fig. 1b; Table 1 and Additional file 1: Figure S1). Thus, the snfl 1 mutant showed multiple morphological defects.

\section{Histological analysis of snfl1 flag leaf}

To investigate the basis for the narrow flag leaf of the snfl1 mutant, we examined the flag leaf anatomy. Compared with the WT, the number of large vascular bundles per flag leaf was reduced by almost half and the number of small vascular bundles per flag leaf was reduced by $43.3 \%$ in the snfll mutant. In addition, the number of small vascular bundles between adjacent large vascular bundles was reduced to $21.3 \%$ of that in the WT (Fig. 1f-l). These results indicate that the narrow flag leaf phenotype should result from the reduced number of vascular bundles in snfll.

To examine the detailed anatomy of the short leaves in snfl1, the epidermis of the leaf was peeled off after cellulase digestion and stained by Toluidine Blue O. The results showed that the number of epidermal cells in the adaxial epidermis of the flag leaf had no obvious difference between the WT and snfl1 mutant. However, the lengths of the epidermal cells of snfl1 were dramatically decreased compared with those of the WT (Fig. 2a-d).

These observations indicate that the decreased length of the epidermal cells and the decreased number of vascular bundles might be associated with the short, narrow flag leaf in the snfl1 mutant. 


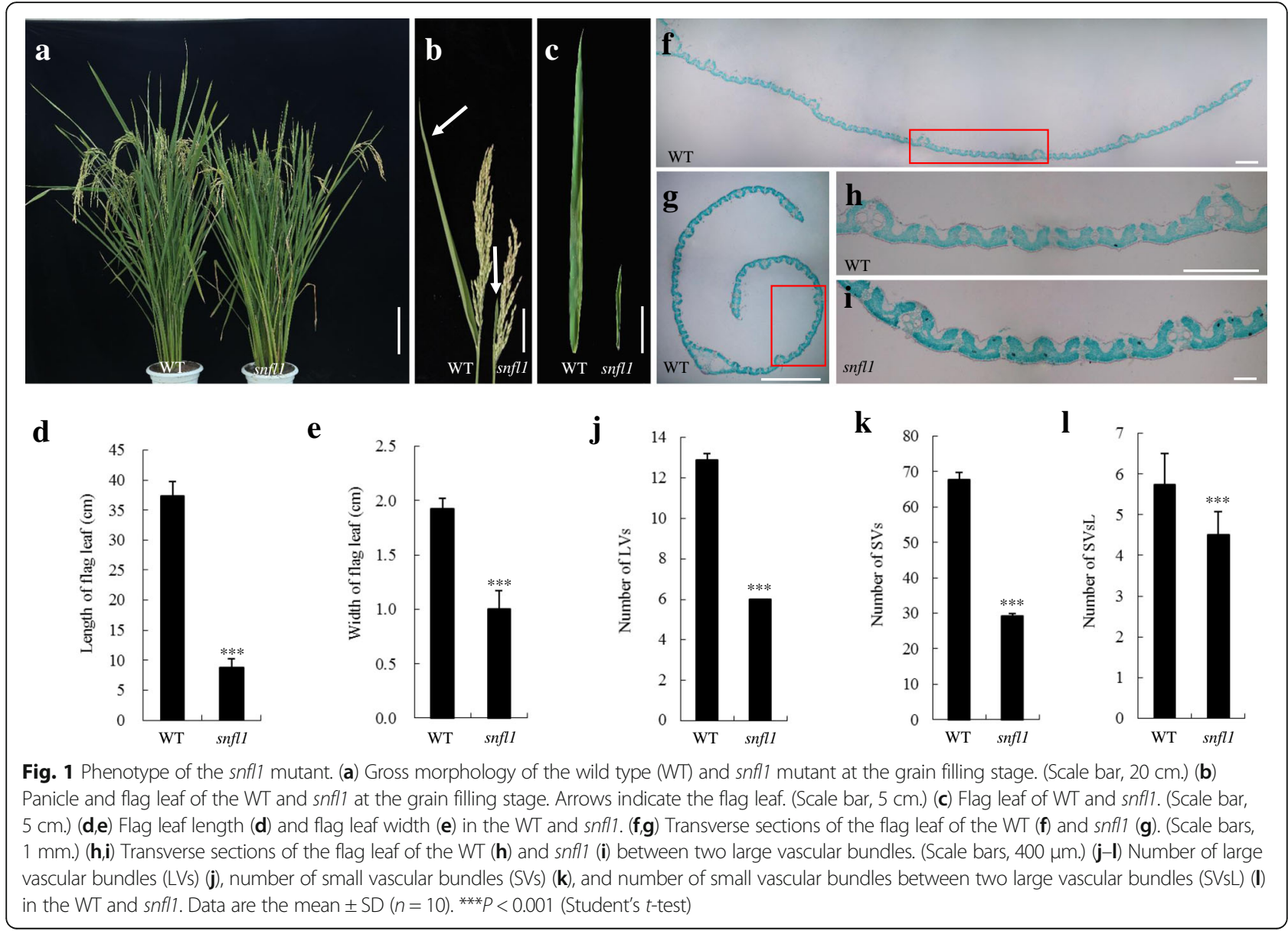

\section{Map-based cloning of SNFL1}

We isolated the SNFL1 gene using a positional cloning method. A genetic population was established from a cross between the snfl1 mutant and japonica rice Nipponbare. All $F_{1}$ hybrids derived from the cross showed a normal phenotype similar to that of Nipponbare. In the

Table 1 Agronomic traits of the wild type (WT) and the snfl1 mutant

\begin{tabular}{lll}
\hline Traits & WT & snfl \\
\hline Plant height (cm) & $117.63 \pm 1.74$ & $118.16 \pm 1.23$ \\
Effective panicle number per plant & $10.00 \pm 1.20$ & $9.33 \pm 1.53$ \\
Panicle length (cm) & $27.10 \pm 0.91$ & $22.76 \pm 1.30^{* * *}$ \\
Number of primary panicle & $11.38 \pm 1.30$ & $8.80 \pm 0.84^{* *}$ \\
Number of second panicle & $38.50 \pm 3.07$ & $28.60 \pm 3.44^{* * *}$ \\
Filled grain number per panicle & $150.00 \pm 16.44$ & $95 \pm 15.38^{* * *}$ \\
Seed setting rate (\%) & $89.01 \pm 1.35$ & $63.79 \pm 0.04^{* * *}$ \\
1000-grain weight (g) & $26.25 \pm 0.48$ & $24.38 \pm 0.68^{* *}$ \\
\hline
\end{tabular}

* means significantly different compared with the corresponding WT at $p<0.01$ and ${ }^{* *}$ means significantly different compared with the corresponding WT at $p<0.001$ by the Student's $t$ test
$\mathrm{F}_{2}$ population, of $4192 \mathrm{~F}_{2}$ individual plants investigated, 980 exhibited the snfl1 mutant phenotype and 3212 exhibited the WT phenotype. The segregation ratio conformed to a 3:1 ratio $\left(x_{0.05}^{2}=2.72<\chi_{0.05}^{2}=3.84\right)$, which indicates that the snfl1 phenotype is controlled by a single recessive nuclear gene. To map the SNFL1 locus, plants showing the snfl1 mutant phenotype were sampled to carry out linkage analysis in the $F_{2}$ population using the bulked segregant analysis method [15]. Rough mapping localized the SNFL1 locus to a 14.25-cM region (about $1.6 \mathrm{Mb}$ ) on the long arm of chromosome 5 delimited by the simple sequence repeat (SSR) markers RM19085 and RM1054 (Fig. 3a). For fine mapping of SNFL1, a suite of Indel and SSR markers were newly designed based on genomic sequence differences between Nipponbare and Jinhui10. Genotyping of $980 \mathrm{~F}_{2}$ mutant individuals revealed that SNFL1 was localized to a 48-kb region between the Indel marker Indel5-3 and the SSR marker RM19157 (Fig. 3a).

According to the Gramene database (http://www.gra mene.org), the 48-kb region contains ten putative open reading frames (ORFs) (Fig. 3b). Among the 10 ORFs 


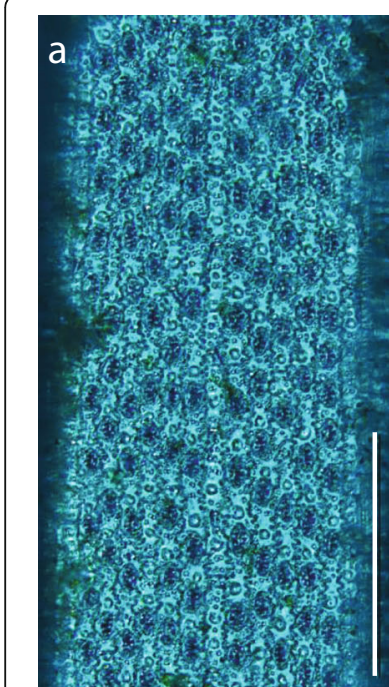

\section{C}

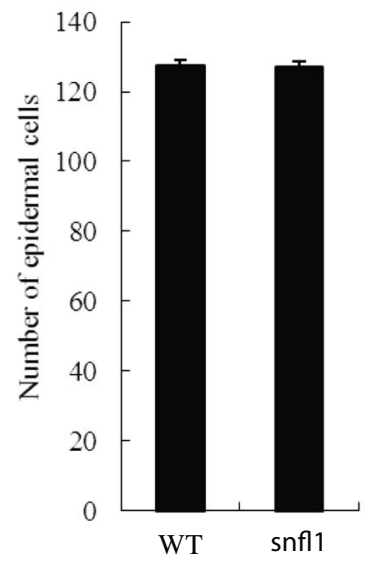

\section{b}

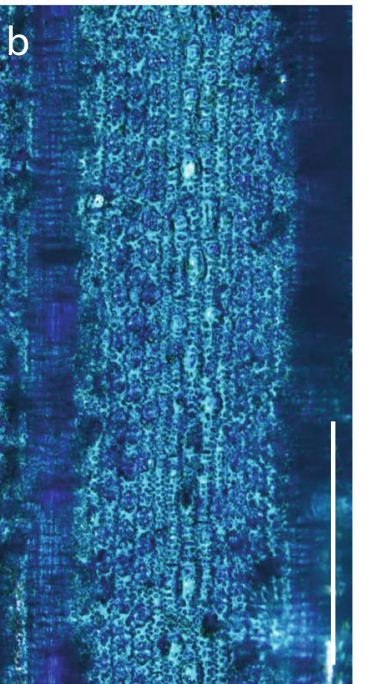

d

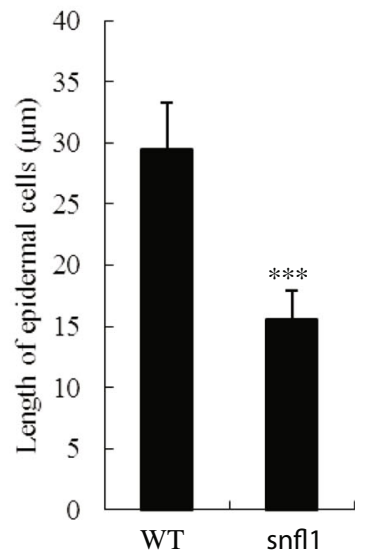

Fig. 2 Histological analysis of the flag leaf. (a) Adaxial epidermal peels of WT mature flag leaves. (Scale bar, $200 \mu \mathrm{m}$.) (b) Adaxial epidermal peels of snfl1 mature flag leaves. (Scale bar, $200 \mu \mathrm{m}$.) (c,d) Number of epidermal cells (c) and length of epidermal cells (d) in the WT and snfli. Means \pm SD are given in $C(n=10)$, and D $(n=60) .{ }^{* *} P<0.001$ (Student's t-test)

sequenced, the snfl1 mutant contained a singlenucleotide transition $(\mathrm{G} \rightarrow \mathrm{A})$, in the only intron at the last base sequence, at the locus LOC_Os05g50270 (Fig. 3c and j). We thus hypothesize that the putative gene LOC_Os05g50270 was the candidate gene of SNFL1. Given that a PstI restriction enzyme cutting site was present at the mutation site (Additional file 2: Figure S2), we carried out enzyme digestion at the mutation site for the mutant and WT plants. The enzyme digestion products were detected by $1 \%$ agarose gel electrophoresis. This analysis demonstrates that the second and third fragment in the mutants could not be digested and resulted in a longer fragment than that of the WT (Fig. 3d-e). This result provides further evidence that the locus LOC_Os05g50270 represents the target gene SNFL1.

The mutation may lead to abnormal splicing of the intron in the snfl 1 mutant. To verify this hypothesis, we sequenced the CDNA of SNFL1 of the WT and snfl1 mutant. Sequence verification of the positive clones identified from the cDNA libraries demonstrated that the WT cDNA contained an 840-bp exon generated by proper splicing of the intron. In contrast, the snfl 1 cDNA contained an 814-bp exon resulting from deletion of 26 bp in second exon (Fig. 3j). The deletion led to early termination of translation. Therefore, the altered intron in snfl1, with the 26-bp deletion in the second exon, is considered to be responsible for the short and narrow flag leaf phenotype in the snfl1 mutant.

To confirm that SNFL1 disruption resulted in the abnormal leaf phenotype, we performed a complementation experiment. The positive plants were detected using a $\beta$-glucuronidase (GUS) array analysis. As expected, the short, narrow leaf phenotype was not observed among the positive plants, all of which showed the normal leaf phenotype. Sequence verification of SNFL1 in the complemented transgenic plants revealed the presence of two peaks at the mutation site (Fig. 3f-i; Additional file 3: Figure S3). These results confirmed that LOC_Os05g50270 corresponds to SNFL1 and the snfl1 mutant harbours defective SNFL1.

\section{Phylogenetic analysis of SNFL1 homologs}

To further clarify the structure and possible function of SNFL1, a bioinformatics analysis was carried out. Multiple-sequence alignment and phylogenetic analysis indicated that SNFL1 consists of 280 amino acids and contains a GATA zinc finger domain. A BLAST search (https://blast.ncbi.nlm.nih.gov/Blast.cgi) revealed that SNFL1 showed high similarity with the conserved GATA zinc finger domain of Hordeum vulgare, Zea mays, Beta vulgaris, and Brassica napus (Fig. $4 \mathrm{a}$ and b). Among the proteins included in the analysis, only TRD1 of Hordeum vulgare has been studied previously. The trd1 mutant fails to suppress bract growth and as a result produces leaf-like structures that subtend each rachis node in the basal portion of the spike, which represents the third outer glume [16]. This phenotype is similar to the snfl 1 mutant, which displayed excessive vegetative growth with overgrown culms from internodes. However, TRD1 has not been cloned, so the underlying molecular mechanism remains unclear.

\section{Analysis of tissue-specific expression of SNFL1}

To examine the expression pattern of SNFL1, we detected SNFL1 transcripts in different WT tissues at different developmental stages using RT-PCR. The abundance of SNFL1 transcripts was highest in the culm. Furthermore, higher quantities of SNFL1 transcripts were detected in 


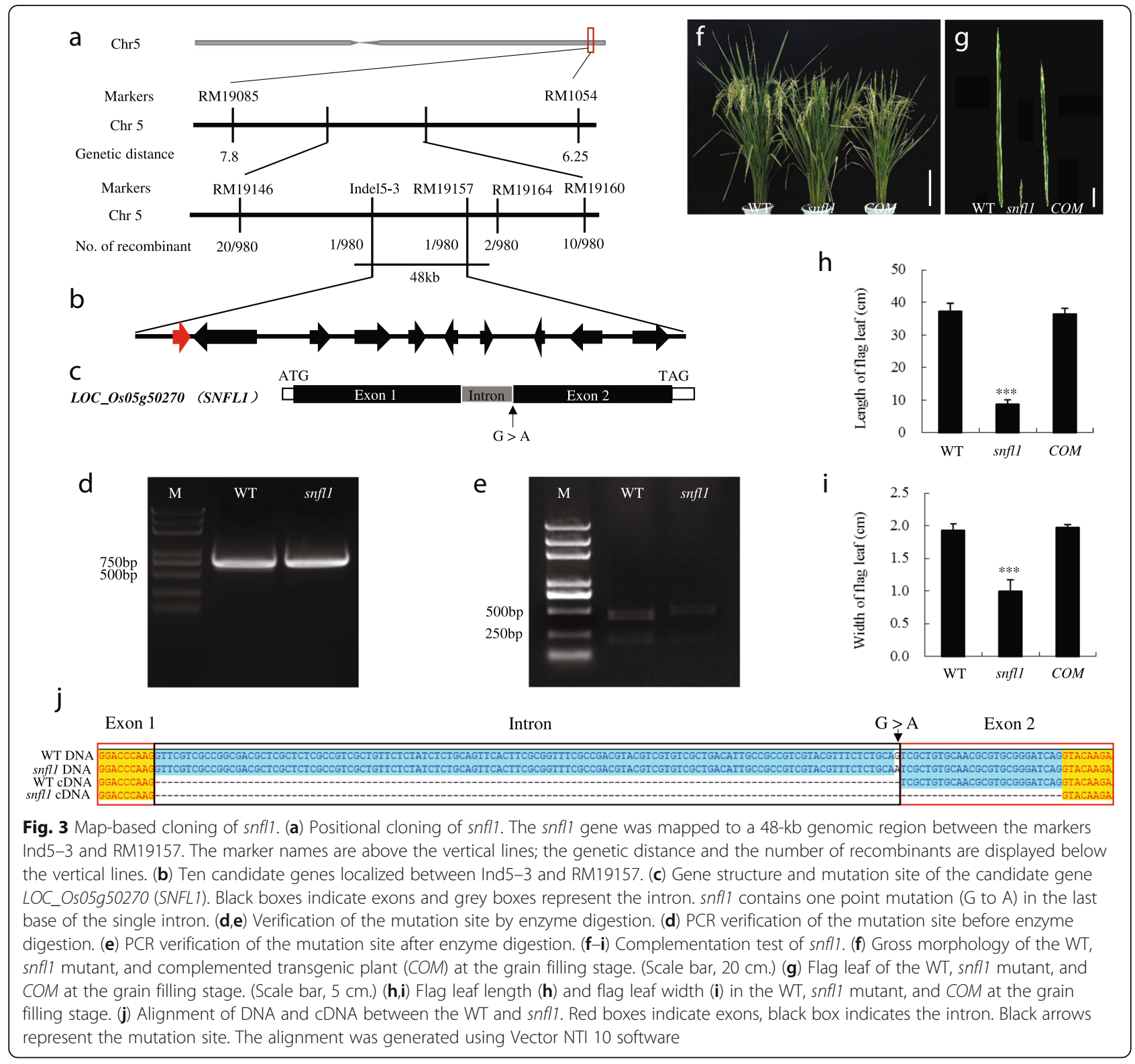

the leaf sheath and root than in other tissues (Fig. 5a). Consistent with this result, promoter-reporter constructs revealed that SNFL1 was predominantly expressed in the culm, leaf sheath and root of transgenic plants (Fig. 5b-h).

\section{Subcellular localization of SNFL1}

To determine the subcellular localization of SNFL1, we fused the green fluorescent protein (GFP) gene to the ORF of SNFL1 driven by the CaMV 35S promoter and transformed the construct into rice protoplasts. The GFP signal was visualized within the protoplast cells, and colocatized with the nucleus-specific stain Nucleus Tracker Red. The co-localization supports the prediction that SNFL1 is a nuclear protein (Fig. 5i).

\section{Discussion}

The short and narrow flag leaves phenotype of snfl1 should result from the abnormal development of epidermal cells and longitudinal veins

Flag leaf length has long been recognized as an important factor that determines plant type for high-yield potential in rice [17]. Elucidation of the genes associated with flag leaf development is essential for clarifying the mechanism that regulates leaf length. However, until recently few genes associated with flag leaf length had been cloned. Analysis of a large number of studies suggests that leaf development is a complex process containing cell expansion and division, axis determination, and tissue differentiation and specification [18]. Thus, cell division and expansion of mesophyll cells, especially 


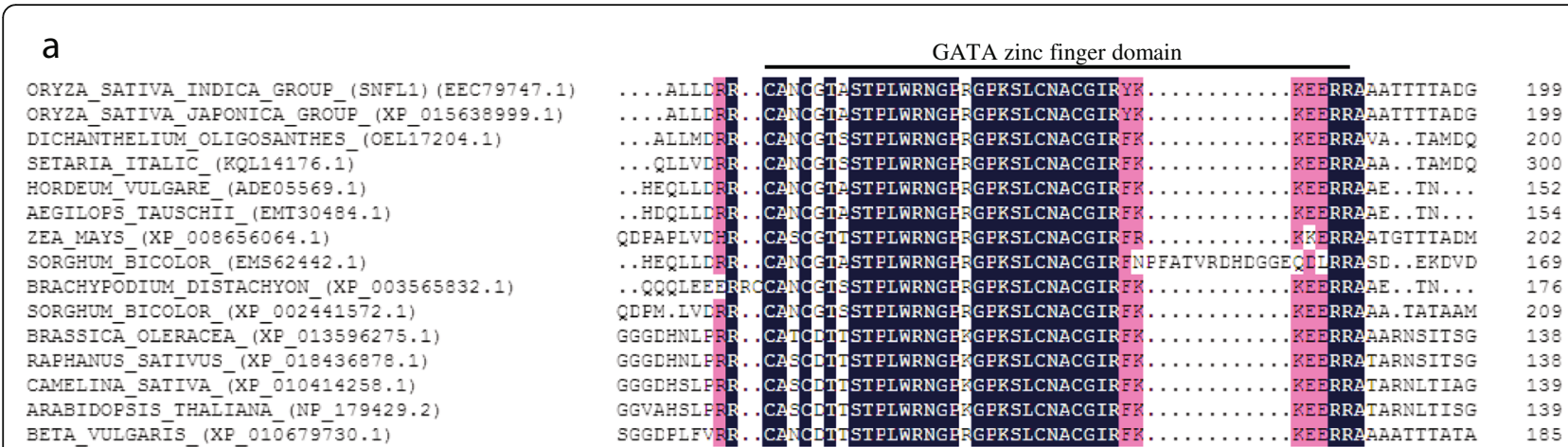

b

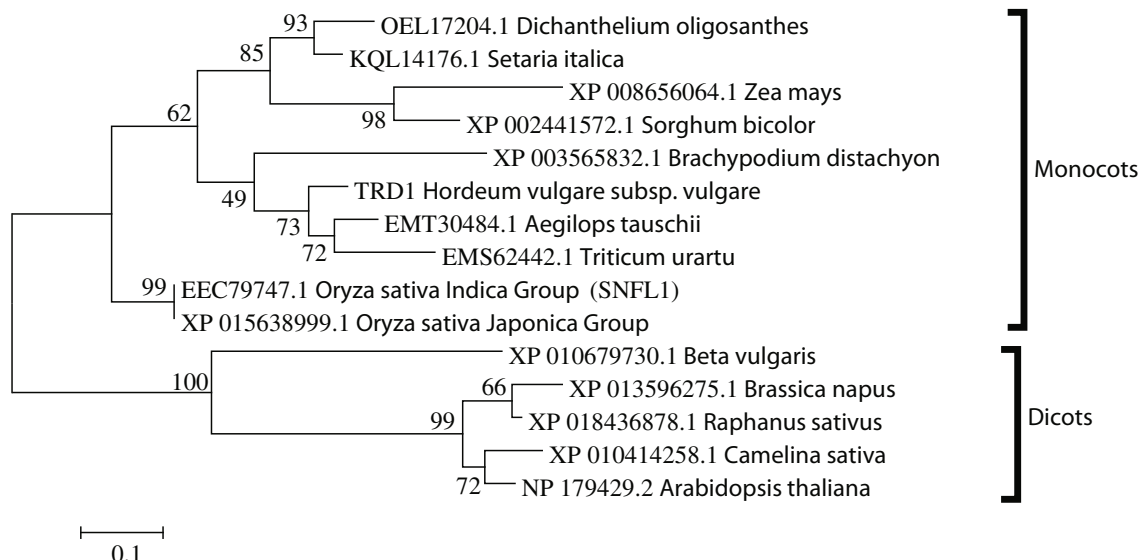

Fig. 4 Multiple-sequence alignment and phylogenetic relationships of SNFL1 with homologous protein sequences. (a) Multiple-sequence alignment of sequences for SNFL1 and other GATA domain-containing proteins. The alignment of GATA domain-containing proteins was generated with ClustalX2.1 software. Dark blue shading indicates 100\% similarity of the aligned sequence; pink shading indicates positions conserved in > 75\% of the aligned sequences. (b) Phylogenetic tree for SNFL1 and GATA domain-containing homologs of high similarity constructed using MEGA 5.05 software

the longitudinal cells, may affect leaf length. In the present study, histological analysis revealed that the length of epidermal cells in the flag leaf of the snfl1 mutant was dramatically decreased compared with that of the WT (Fig. 2a-d). Therefore, the short flag leaf phenotype of snfl1 may result from abnormal development of the epidermal cells.

Narrow flag leaves result mainly from reduced lateral-axis outgrowth and fewer longitudinal veins in rice $[11,19]$. A number of genes associated with the development of longitudinal veins in rice have been studied. For example, the nal1 mutant exhibits a significant phenotype of narrow leaves. In accordance with the narrow leaves, nal1 leaves contain a decreased number of longitudinal veins. NAL1 affects vascular pattern of rice and polar auxin transport, and plays an important role in lateral leaf growth [10]. In addition, the narrow leaf phenotypes of the nal2, nal3, nrl1, nrl2, and $a v b$ mutants may be due to reduced lateral-axis outgrowth with fewer longitudinal veins $[11,13,20]$. In the present study, the snfl 1 mutant phenotype also involved changes to the longitudinal veins. The number of longitudinal veins was distinctly decreased in the flag leaf of the snfl1 mutant compared with that of the WT (Fig. 1c and e). The alteration in number of longitudinal veins in the flag leaf may indicate that SNFL1 is associated with the development of longitudinal veins. However, the snfl 1 mutant also showed reduced panicle length, number of panicles, and 1000-grain weight, which indicates that SNFL1 has a complicated function in rice plant development.

\section{Alternative splicing of SNFL1 might result in short and narrow flag leaves in snfl1}

In eukaryotes, most encoding genes are split genes that contain introns. At least $74 \%$ multi-exon genes of human are alternatively spliced and may comprise $80-85 \%$ of such genes $[21,22]$. During the process of gene expression in animals and plants, introns of split genes are excised under precise regulation, and subsequently the exons are spliced to form the mature mRNA, which is translated into a functional protein. The first splicing factor identified in plants was AtRSP31, which contains 


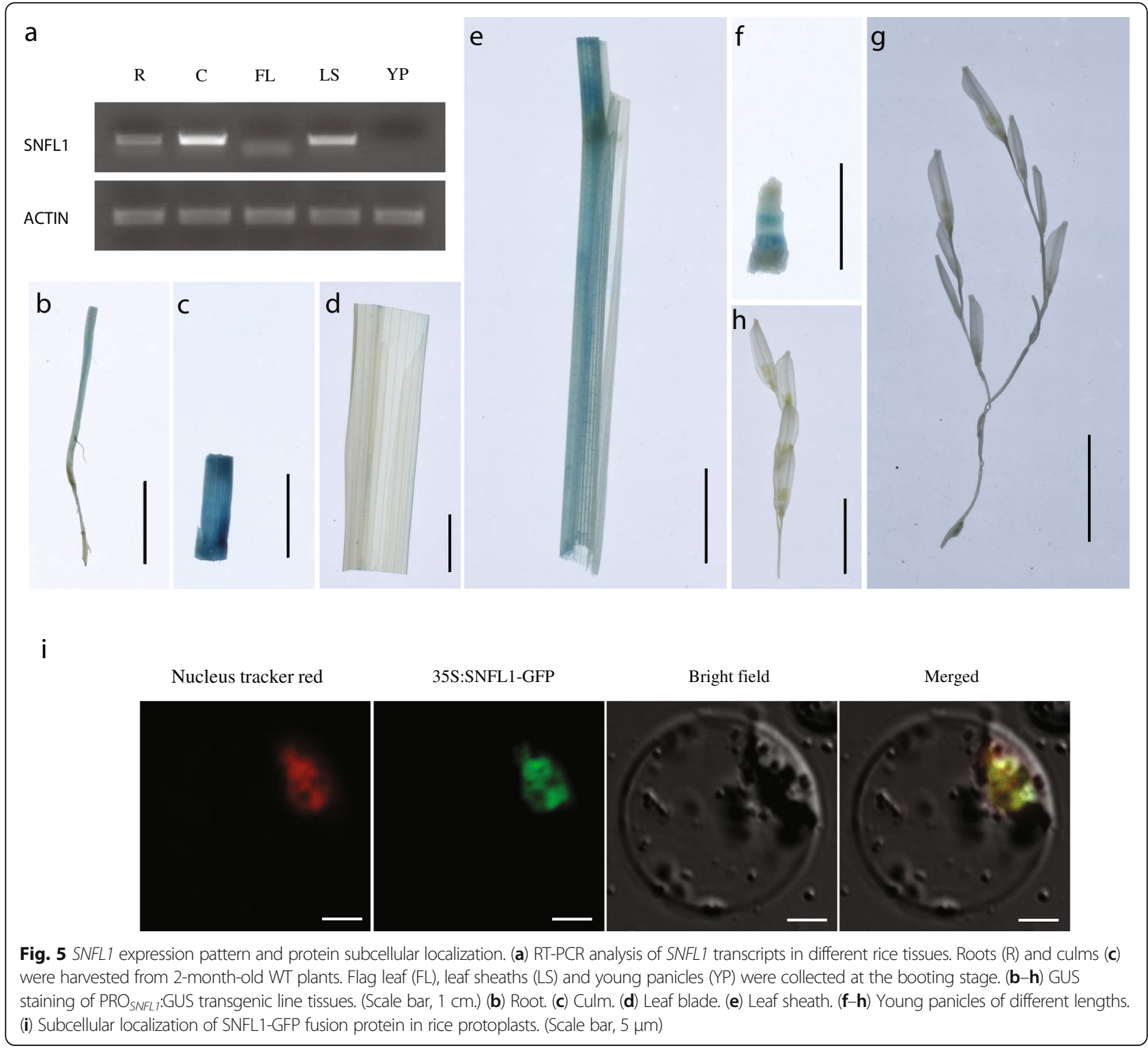

an N-terminal RNA recognition motif and a C-terminal RS domain highly enriched in arginines. As a true plant splicing factor, AtRSP31 plays an important role in splicing, similar to that of other RS splicing factors. RS domain splicing factors play crucial roles in alternative and constitutive splicing in plants [23]. An additional typical splicing factor gene is Waxy in rice. $W x^{a}$ and $W x^{b}$ are two functional alleles of the rice waxy $(w x)$ locus, they are defined by the huge difference in the amount of the gene product, called $\mathrm{Wx}$ protein, that accumulates in mature grains. The $W x^{a}$ allele has a normal sequence of GT at the $5^{\prime}$ splice junction of the first intron and encodes a high proportion of the $W x$ transcripts in the endosperm, whereas the $W x^{b}$ allele has TT at the $5^{\prime}$ splice junction and shows a low expression level among the mature transcripts. The low expression level of $W x^{b}$ is a result of a single-base mutation at the $5^{\prime}$ splice site of the first intron. Northern blot analysis also indicates that the larger transcript consisting of the unspliced first intron is closely related to the function of the $W x^{b}$ allele. The $W x^{b}$ allele of rice carrying the G-to-T mutation of the first intron has been conserved in cultivated rice because the mutation causes low amylose content in the grain [24, 25]. Thus, mutation at splice sites of introns always leads to alternative splicing (AS), which is a crucial mechanism of post-transcriptional regulation, and can increase mRNA stability and protein diversity. Furthermore, AS plays a fundamental role in plant development, growth, and responses to external cues. The instances of AS identified in flowering plants far exceeds previous predictions, and thus its function in plants may also surpass expectations [26]. 
The identification of the mRNA splicing site is mainly accomplished via splicing complexes, of which there are two main kinds in higher eukaryotes. The first is the dominant U2 type splicing complex, which contains several small intranuclear complexes of U1, U2, U4, U5, and U6. The function of the U2 type splicing complex is to cut the intron of the GT-AG type splicing site. The second is the U12 type splicing complex, including several small intranuclear complexes of U11, U12, U4A, U5, and U6. The function of the U12 type splicing complex is to cut a small part of the introns $[27,28]$. The introns in plants are rich in $\mathrm{U}$ and $\mathrm{UA}$, and the exons are rich in G. This bias is crucial for the identification of the splicing sites and the efficiency of the splicing. The specific splicing mechanism remains unclear, but it is now certain that four splicing recognition signals play an important role in the normal splicing of the pre-mRNA introns. These signals are: (1) The conserved recognition sequence at the 5 ' terminal of the intron is GU. (2) The conserved recognition sequence at the 3 ' terminal of the intron is AG, specifically recognizable by the small nuclear U2 subunit. (3) The polypyrimidine recognizable by the small nuclear protein U2 factor AF65 at the 3' terminal of the intron. (4) The key branch locus, the sequence features of which are conserved CURAY ( $\mathrm{R}$ is purine, $\mathrm{Y}$ is pyrimidine), its position being about 17 40 nucleotides away from the $3^{\prime}$ terminal splicing site, which can be seen from the small nucleoprotein U2 Subunit $[29,30]$. In the present study, we found that the intron of SNFL1 conforms to most of the splicing rules, starting with GU and ending with AG, and the $3^{\prime}$ terminal can be recognized by the U2 subunit. It is worth noting that the $3^{\prime}$ terminal sequence of the exon fragment that is cut off is also AG, therefore the reason for the AS of snfl1 may be the 3' terminal mutation of the intron, making it unrecognizable by the U2 subunit. The next -AG sequence is recognizable in the 3' terminal sequence of the exon that was cut off (Fig. 3j). The short and narrow flag leaf phenotype of snfll may result from AS of the intron, and the snfll mutant may thus provide valuable material for future research on alternative splicing.

\section{Role of GATA transcription factors in plant development}

Transcription factors serve multiple functions. They can be combined with the target gene promoter and can affect positive or negative regulation of transcriptional activity. Many families of transcription factors are known in plants, such as the bZIP, bHLH, FAR1, CAMTA, GRAS, NAC, and GATA families. The GATA family has the ability to recognize GATA motifs, and most contain zinc finger structures. The GATA family is characterized by an extremely high affinity for the consensus sequence (T/A)GATA(A/G) and have been identified among fungi, metazoans, and plants [31]. GATA is a type of transcription factor that is prevalent in eukaryotes and plays an important role in biological processes, such as regulation of plant light response, chlorophyll synthesis, cytokinin response, and metabolism of carbon and nitrogen. The (T/A)GATA(A/G) sequence was first detected in the chicken globin gene promoter [32]. Subsequently, the transcription factor GATA-1 was identified in 1991, followed by the isolation of other GATA transcription factors [33-36]. GATA transcription factors are members of the zinc finger protein family, and can identify and specifically bind DNA sequences. The DNA binding domain of GATA factors constitutes a type IV zinc finger in the form of $\mathrm{CX}_{2} \mathrm{CX}_{17-20} \mathrm{CX}_{2} \mathrm{C}$, followed by a highly basic region [31]. This is consistent with the present finding that SNFL1 contains a GATA zinc finger domain of the form $\mathrm{CX}_{2} \mathrm{CX}_{18} \mathrm{CX}_{2} \mathrm{C}$ (Fig. 4a).

GATA transcription factors are common in plants and play an important role in regulation of flowering time, leaf growth, flower development, photoperiodism and optical signal transduction. These biological processes are associated with growth and development of plants. For example, the $C O$ gene was suggested to encode a protein with two zinc fingers related to those of GATA transcription factors. $C O$ is associated with circadian rhythm regulation and regulation of meristems, and therefore plays an important role in regulation of flowering time by adjusting the photoperiod [37]. The snfl1 mutant produces short and narrow leaves, which indicates that SNFL1 may play an important role in rice leaf development. Some GATA transcription factors have been identified previously in rice. One example is NECK LEAF1 (NL1), the allelic gene of SNFL1, in which the nl1 mutant shows abnormal patterns of upper internode elongation, smaller panicles with overgrown bracts, and a delayed flowering time. Overexpression of NL1 in transgenic plants usually results in severe growth retardation, smaller leaves, and fewer vegetative phytomers, which indicates that NL1 plays an important role in organ differentiation [38]. In the present study, snfl1 has overgrown culms and abnormal panicles (Fig. $1 \mathrm{~b}$ and Additional file 1: Figure S1), which is similar to $n l 1$ and may indicate that the functions of NL1 and SNFL1 have much in common. On the whole, SNFL1 encodes a GATA zinc finger protein and may have multiple functions in plant development.

\section{Conclusions}

In this study, a gene that affects leaf length and width, which we term short and narrow flag leaf 1 (SNFL1), was identified in rice using a map-based cloning strategy. The gene is located in a 48-kb genomic region between Indel53 and RM19157 on chromosome 5. Sequencing analysis revealed the presence of a single-nucleotide mutation $(\mathrm{G} \rightarrow \mathrm{A})$ at the locus LOC_Os05g50270. LOC_Os05g50270 was confirmed to correspond with SNFL1 by transgenic complementation. SNFL1 encodes a GATA zinc finger domain-containing protein and its loss of function led to 
development of short and narrow leaves. The short and narrow leaf phenotype was associated with abnormal development of the epidermal cells and longitudinal veins. SNFL1 might have functions in multiple biological processes, such as the development of epidermal cells, longitudinal veins, and panicle development.

\section{Methods}

\section{Plant material}

The snfl1 mutant with short flag leaf was isolated from the $\mathrm{M}_{2}$ generation of indica rice (Oryza sativa L. subsp. indica) 'Jinhui 10 ' by EMS treatment. The $\mathrm{F}_{2}$ mapping population was raised from a cross between the sufl1 mutant and japonica rice (O. sativa subsp. japonica) 'Nipponbare'. All plants were grown under natural conditions in an experimental field at the Southwest University Rice Research Institute in Chongqing, China.

\section{Histological analysis}

The middle portion of flag leaf of WT and snfl1 plants at the heading stage was fixed in FAA solution (70\% ethanol, $5 \%$ formaldehyde and $5 \%$ acetic acid $[v / v / v]$ ) for $48 \mathrm{~h}$. The samples were infiltrated and embedded in paraffin after dehydration in an ethanol series. The embedded samples were cut into sections $8 \mu \mathrm{m}$ thick with a rotary microtome (RM2245, Leica Microsystems, Hamburg, Germany), stained with fast green and counterstained with safranin, and observed under a light microscope (Eclipse Ci-L, Nikon, Tokyo, Japan).

For anatomical observation of the leaf epidermis, about $1 \mathrm{~cm}$ from the middle portion of rice leaves was excised, placed under water, and evacuated with a vacuum pump for $30 \mathrm{~min}$. The evacuated samples were digested for $48 \mathrm{~h}$ in $2 \%$ cellulase R-10, and stained overnight in $0.5 \%$ toluidine blue $\mathrm{O}$. After washing with water, an epidermal peel was prepared and sealed under a coverslip for observation with a light microscope (Eclipse Ci-L, Nikon, Tokyo, Japan) and digital images were captured following the method of Li et al. [39].

\section{Measurement of agronomic traits}

Five WT and five snfl 1 mutant plants were used to measure agronomic traits at the mature stage. The measured agronomic traits comprised the length and width of three functional leaves, plant height, effective panicle number per plant, panicle length, number of primary panicles, number of secondary panicles, number of filled grains per panicle, seed setting rate, and 1000-grain weight. Values presented are the means \pm SD of three biological repeats. The student's $t$-test was applied for statistical analysis of the data.

\section{DNA extraction and PCR analysis}

Total genomic DNA was extracted from fresh leaves using the cetyl trimethylammonium bromide method
[40] at the heading stage once visibly distinct phenotypes were apparent. The total PCR reaction volume was $12.5 \mu \mathrm{L}$ and contained the following components: $1.25 \mu \mathrm{L}$ of $10 \times$ PCR buffer, $1 \mu \mathrm{L}$ of $10 \mu \mathrm{mol} \mathrm{L}^{-1}$ primers, $1 \mu \mathrm{L}$ of $50 \mathrm{ng} \mu \mathrm{L}^{-1} \mathrm{DNA}, 0.75 \mu \mathrm{L}$ of $25 \mathrm{mmol} \mathrm{L}^{-1}$ $\mathrm{MgCl}_{2}, 0.5 \mu \mathrm{L}$ of $2.5 \mathrm{mmol} \mathrm{L}{ }^{-1}$ dNTPs, $7.9 \mu \mathrm{L} \mathrm{ddH}_{2} \mathrm{O}$, and $0.1 \mu \mathrm{L}$ of $5 \mathrm{U} \mathrm{LL}^{-1}$ Taq DNA polymerase. The PCR reaction system was as follows: $5 \mathrm{~min}$ at $94{ }^{\circ} \mathrm{C}$, followed by 35 cycles of $30 \mathrm{~s}$ at $94{ }^{\circ} \mathrm{C}, 30 \mathrm{~s}$ at $55^{\circ} \mathrm{C}$ and $1 \mathrm{~min}$ at $72{ }^{\circ} \mathrm{C}$. The PCR products were separated in a $10 \%$ polyacrylamide gel and visualized by rapid silver staining.

\section{Development of new Indel and SSR markers}

To localize SNFL1 to a narrower chromosomal interval, new Indel and SSR markers were designed because of the lack of known markers. The japonica rice Nipponbare genome sequence [1] was used as a query for a BLAST search against the entire genome sequence of indica rice Jinhui10. Differences in more than two nucleotides were chosen to design Indel markers, and new SSR markers were identified on the basis of differences in the length of repeat sequences of Nipponbare and Jinhui10 using SSR-Hunter 1.3. The newly designed markers are listed in Additional file 4: Table S1.

\section{Map-based cloning of SNFL1}

To determine the chromosomal location of SNFL1, $980 \mathrm{~F}_{2}$ plants that showed the mutant phenotype were selected and used as a mapping population. Initial mapping was carried out using SSR markers based on 12 rice linkage maps (http://rgp.dna.affrc.go.jp). Fine mapping was performed using the newly designed Indels and SSR markers after the chromosomal region was determined by linkage analysis. The sequences of primers used in the mapping are listed in Additional file 4: Table S1.

\section{Vector construction}

To construct the SNFL1 complementation plasmid, a 3881-bp genomic DNA fragment containing the entire 960 bp SNFL1 coding sequence, a 2362-bp upstream region, and a 559-bp downstream region were amplified using the primers SNFL1com-F and SNFL1com-R from WT genomic DNA. The amplified fragment was digested with BamHI and HindIII, and cloned into the binary vector pCAMBIA1301. The recombinant plasmids were introduced into the snfl 1 mutant by Agrobacterium tumefaciens-mediated transformation as described previously [41]. The primer sequences are listed in Additional file 5: Table S2.

\section{GUS expression assay}

To analyse the expression pattern of SNFL1, a 3031-bp promoter fragment was cloned into the pCAMBIA1301 vector using the SNFL1-specific primers SNFL1-PF and SNFL1-PR to create the $P R O_{S N F L 1}: G U S$ reporter gene 
construct, which was then transformed into Jinhui10 by A. tumefaciens-mediated transformation. GUS staining was performed on $P R O_{S N F L 1}$ :GUS $\mathrm{T}_{1}$ generation transgenic plants in accordance with the method of Jefferson et al. [42]. Digital images were captured using a Canon EOS 5D Mark III camera. Primers used to clone the promoter fragment are listed in Additional file 5: Table S2.

\section{Subcellular localization}

The coding sequence of SNFL1 lacking the stop codon was fused to the $\mathrm{N}$-terminus of the GFP gene under the control of the enhanced CaMV 35S promoter in the SpeI and $B a m H I$ sites of the vector pAN580 to generate the pSNFL1-GFP construct. The SNFL1-GFP fusion protein was transiently expressed in rice leaf protoplasts by a previously described method [14]. Fluorescence was observed using a LSM800 confocal laser microscope (Zeiss, Jena, Germany). The primer sequences used are listed in Additional file 5: Table S2.

\section{Protein sequence and phylogenetic analysis}

For phylogenetic analysis of SNFL1 homologs in plants, protein sequences were obtained by searching GenBank (http://www.ncbi.nlm.nih.gov/genbank/) using the SNFL1 sequence as a query. A multiple-sequence alignment was generated using ClustalX2.1. A phylogenetic tree was constructed with MEGA 5.05 using the maximum likelihood method. Topological robustness was assessed by means of a bootstrap analysis with 1000 replicates.

\section{Additional files}

Additional file 1: Figure S1. The phenotype of snfl1 mutant. (A-B) Plants of WT and snfl1 in the field. (C) A culm of WT and snfl1 mutant at maturing stage, vegetative leaves being removed. Arrow indicate overgrown culms. (Scale bar, $10 \mathrm{~cm}$.) (D) Internodes and panicles of WT and snfl1 mutant. (Bars $=5 \mathrm{~cm}$.) (E) Statistical data of internodes in WT and snfl1. (F-G) Statistical data of length and width of second and third leaves between $\mathrm{WT}$ and snfl1. Means $\pm \mathrm{SD}$ are given in $\mathrm{E}(n=10), \mathrm{F}(n=50)$, and $\mathrm{G}(n=50)$. ${ }^{* *} P<0.01,{ }^{* * *} P<0.001$ (t-test). (PPT $1000 \mathrm{~kb}$ )

Additional file 2: Figure S2. Genetic sequences of LOC_Os05950270. The ATG and TAG codon were shaded in green, the mutant site was shaded in grey. The red box indicates the restriction enzyme cutting site at the mutant site. The primer sequences to sequence CDNA were shaded in yellow. (PPT $153 \mathrm{~kb}$ )

Additional file 3: Figure S3. Sequence verification of SNFL1 in complement transgenic plants. Black arrows represent the peak of mutation and rescue. (PPT $96 \mathrm{~kb}$ )

Additional file 4: Table S1. Primer sequences for mapping SNFL1. (XLS $28 \mathrm{~kb}$ )

Additional file 5: Table S2. Primers used to construct vectors. (XLS $26 \mathrm{~kb}$ )

\section{Abbreviations}

AS: Alternative splicing; BLAST: Basic Local Alignment Search Tool; COM: Complemented transgenic plant; EMS: Ethyl methanesulfonate; GFP: Green fluorescent protein; GUS: $\beta$-glucuronidase; IRGSP: International Rice Genome Sequencing Project; ORFs: Open reading frames; RTPCR: Reverse transcription-polymerase chain reaction; SSR: Simple sequence repeat; WT: Wild type

\section{Acknowledgements}

We gratefully acknowledge the assistance of Nanjing Agriculture University for providing the vector of pAN580, the nucleus:mCherry marker and the PEG-mediated rice protoplast transformation method.

\section{Funding}

This work was supported by grants from the Ministry of Science and Technology of China (Grants 2016YFD0100501), National Transgenic Major Program of China (2016ZX08001-002), Chongqing Science and Technology Innovation Special Project (cstc2016shms-ztzx80007, cstc2017shmsxdny80057), Chongqing Research Program of Basic Research and Frontier Technology (cstc2015jcyjA80008) and Fundamental Research Funds for the Central Universities (XDJK2015D031)

\section{Availability of data and materials}

The datasets generated and analysed during the current study are available from the corresponding author on reasonable request.

\section{Authors' contributions}

HG, SX, WX, and HP planned and designed the research. HP, JY, ZXB, TW, and ZXQ performed the experiments. SX, HP, ZXB, TW, LY, S Y, XJ and NJ conducted the fieldwork. HP, SX and ZXB analyzed the data. HP and SX wrote the manuscript. All authors have read and approved the manuscript for publication.

\section{Ethics approval and consent to participate}

The field work in our study was approved by local legislation.

Consent for publication

Not applicable.

\section{Competing interests}

The authors declare that they have no competing interests.

\section{Publisher's Note}

Springer Nature remains neutral with regard to jurisdictional claims in published maps and institutional affiliations.

\section{Author details}

${ }^{1}$ Key Laboratory of Application and Safety Control of Genetically Modified Crops, Rice Research Institute of Southwest University, Academy of Agricultural Sciences, Southwest University, Chongqing, China. ${ }^{2}$ Key Laboratory of Southwest Rice Biology and Genetic Breeding, Institute of Rice and Sorghum, Sichuan Academy of Agricultural Sciences, Deyang, China.

Received: 29 October 2017 Accepted: 27 September 2018

Published online: 09 November 2018

References

1. Project IRGS. The map-based sequence of the rice genome. Nature. 2005; 436(7052):793-800.

2. Ghosh S, Sahai VN, Saran S. Role of flag leaf on grain yield and spikelet sterility in rice cultivar. Oryza. 1990;27:87-9.

3. Jebbouj R, Yousfi BE. Barley yield losses due to defoliation of upper three leaves either healthy or infected at boot stage by Pyrenophora teres $f$. teres. Eur J Plant Pathol. 2009;125(2):303-15.

4. Li ZK, Pinson SRM, Stansel JW, Paterson AH. Genetic dissection of the source-sink relationship affecting fecundity and yield in rice (Oryza sativa L.). Mol Breed. 1998;4(5):419-26.

5. Monyo $\mathrm{JH}$, Whittington WJ. Genotypic differences in flag leaf area and their contribution to grain yield in wheat. Euphytica. 1973;22(3):600-6.

6. Komorisono M. Analysis of the Rice mutant dwarf and gladius leaf 1 . Aberrant Katanin-mediated microtubule organization causes up-regulation of gibberellin biosynthetic genes independently of gibberellin signaling. Plant Physiol. 2005;138(4):1982-93.

7. Ji SH, Gururani MA, Lee JW, Ahn BO, Chun SC. Isolation and characterisation of a dwarf rice mutant exhibiting defective gibberellins biosynthesis. Plant Biol. 2014;16(2):428-39.

8. Liu GM, Zhang K, Ai J, Deng XJ, Hong YY. Wang XM. Patatin-related phospholipase a, pPLAllla, modulates the longitudinal growth of vegetative tissues and seeds in rice. J Exp Bot. 2015;66(21):6945-55. 
9. Mori M, Nomura T, Ooka H, Ishizaka M, Yokota T, Sugimoto K, et al. Isolation and characterization of a Rice dwarf mutant with a defect in Brassinosteroid biosynthesis. Plant Physiol. 2002;130(3):1152-61.

10. Qi J, Qian Q, Bu QY, Li SY, Chen Q, Sun JQ, et al. Mutation of the Rice Narrow leaf1 gene, which encodes a novel protein, affects vein patterning and polar auxin transport. Plant Physiol. 2008;147(4):1947-59.

11. Cho SH, Yoo SC, Zhang HT, Pandeya D, Koh HJ, Hwang JY, et al. The rice narrow leaf2 and narrow leaf3 loci encode WUSCHEL-related homeobox 3A (OsWOX3A) and function in leaf, spikelet, tiller and lateral root development. New Phytol. 2013;198(4):1071-84.

12. Fujino K, Matsuda Y, Ozawa K, Nishimura T, Koshiba T, Fraaije MW, et al. NARROW LEAF 7 controls LEAF shape mediated by auxin in rice. Mol Gen Genomics. 2008:279(5):499-507.

13. Hu J, Zhu L, Zeng DL, Gao ZL, Guo LB, Fang YX, et al. Identification and characterization of NARROW AND ROLLED LEAF 1, a novel gene regulating LEAF morphology and plant architecture in rice. Plant Mol Biol. 2010;73(3):283-92.

14. Ma L, Sang XC, Zhang T, Yu ZY, Li YF, Zhao FM, et al. ABNORMAL VASCULAR BUNDLES regulates cell proliferation and procambium cell establishment during aerial organ development in rice. New Phytol. 2017:213(1):275-86.

15. Michelmore RW, Paran I, Kesseli RV. Identification of markers linked to disease-resistance genes by bulked segregant analysis: a rapid method to detect markers in specific genomic regions by using segregating populations. Proc Natl Acad Sci U S A. 1991;88(21):9828-32.

16. Houston K, Druka A, Bonar N, Macaulay M, Lundqvist U, Franckowiak J, et al. Analysis of the barley bract suppression gene Trd1. Theor Appl Genet. 2012; 125(1):33-45.

17. Yuan LP. Hybrid rice breeding for super high yield. Hybrid Rice. 1997;12(6):1-6.

18. Itoh Jl, Nonomura Kl, Ikeda K, Yamaki S, Inukai Y, Yamagishi H, et al. Rice Plant development: from zygote to spikelet. Plant Cell Physiol. 2005;46(1):23-47.

19. O'Toole JC, Cruz RT. Response of leaf water potential, stomatal resistance, and leaf rolling to water stress. Plant Physiol. 1980;65(3):428-32.

20. Zhao SS, Zhao L, Liu FX, Wu YZ, Zhu ZF, Sun CQ, et al. NARROW AND ROLLED LEAF 2 regulates LEAF shape, male fertility, and seed size in rice. J Integr Plant Biol. 2016:58(12):983-96.

21. Sharp PA, Burge CB. Classification of introns: U2-type or U12-type. Cell. 1997; 91(7):875-9.

22. Johnson JM, Castle J, Garrett-Engele P, Kan Z, Loerch PM, Armour CD, et al. Genome-wide survey of human alternative pre-mRNA splicing with exon junction microarrays. Science. 2003;302(5653):2141-4.

23. Lopato S, Waigmann E, Barta A. Characterization of a novel arginine/serinerich splicing factor in Arabidopsis. Plant Cell. 1996;8(12):2255-64.

24. Hirano HY, Eiguchi M, Sano Y. A single base change altered the regulation of the waxy gene at the posttranscriptional level during the domestication of rice. Mol Biol Evol. 1998;15(8):978-87.

25. Isshiki M, Morino K, Nakajima M, Okagaki RJ, Wessler SR, Izawa T, et al. A naturally occurring functional allele of the rice waxy locus has a GT to $\Pi T$

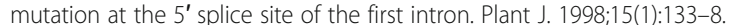

26. Luco RF, Allo M, Schor IE, Kornblihtt AR, Misteli T. Epigenetics in alternative pre-mRNA splicing. Cell. 2011;144(1):16-26.

27. Lorkovic ZJ, Kirk DAW, Lambermon MHL, Filipowicz W. Pre-mRNA splicing in higher plants. Trends Plant Sci. 2000;5(4):160-7.

28. Luehrsen KR, Taha S, Walbot V. Nuclear pre-mRNA processing in higher plants. Prog Nucleic Acid Res Mol Biol. 1994:47:149-93.

29. Brown JWS, Simpson CG. Splice site selection in plant pre-mRNA splicing. Annu Rev Plant Phys. 1998:49:77-95.

30. Wu Q, Krainer AR. AT-AC pre-mRNA splicing mechanisms and conservation of minor introns in voltage-gated ion channel genes. Mol Cell Biol. 1999; 19(5):3225-36.

31. Reyes JC, Muro-Pastor MI, Florencio FJ. The GATA family of transcription factors in Arabidopsis and Rice. Plant Physiol. 2004;134(4):1718-32.

32. Evans T, Rettman M, Felsenfeld G. An erythrocyte-specific DNA-binding factor recognizes a regulatory sequence common to all chicken globin genes. Proc Natl Acad Sci U S A. 1988:85(16):5976-80.

33. Pevny L, Simon MC, Robertson E, Klein WH, Tsai SF, D'Agati V, et al. Erythroid differentiation in chimaeric mice blocked by a targeted mutation in the gene for transcription factor GATA-1. Nature. 1991;349(17):257-60.

34. Tsal FY, Keller G, Kuo FC, Welss M, Chen J, Rosenblatt M, et al. An early haematopoietic defect in mice lacking the transcription factor GATA-2. Nature. 1994;371(6494):221-6.

35. Zheng W, Flavell RA. The transcription factor GATA-3 is necessary and sufficient for Th2 cytokine gene expression in CD4 T cells. Cell. 1997;89(4):587-96.
36. Molkentin JD. The zinc finger-containing transcription factors GATA-4, -5 , and -6. J Biol Chem. 2000:275(50):38949-52.

37. Putterill J, Robson F, Karen Lee RS, Coupland G. The CONSTANS gene of Arabidopsis promotes flowering and encodes a protein showing similarities to zinc finger transcription factors. Cell. 1995;80(6):847-57.

38. Wang LP, Yin HF, Qian Q, Yang J, Huang CF, Hu XH, et al. NECK LEAF 1, a GATA type transcription factor, modulates organogenesis by regulating the expression of multiple regulatory genes during reproductive development in rice. Cell Res. 2009;19(5):598-611.

39. Li L, Shi ZY, Li L, Shen GZ, Wang XQ, An LS, et al. Overexpression of ACL1 (abaxially curled leaf 1) increased Bulliform cells and induced Abaxial curling of leaf blades in Rice. Mol Plant. 2010;3(5):807-17.

40. Murray MG, Thompson WF. Rapid isolation of high molecular weight plant DNA. Nucleic Acids Res. 1980;8(19):4321-6.

41. Sang XC, Li YF, Luo ZK, Ren DY, Fang LK, Wang N, et al. CHIMERIC FLORAL ORGANS1, encoding a monocot-specific MADS box protein, regulates Floral organ identity in Rice. Plant Physiol. 2012;160(2):788-807.

42. Jefferson RA, Kavanagh TA, Bevan MW. GUS fusions: $\beta$-glucuronidase as a sensitive and versatile gene fusion marker in higher plants. EMBO J. 1987; 6(13):3901-7.

\section{Ready to submit your research? Choose BMC and benefit from:}

- fast, convenient online submission

- thorough peer review by experienced researchers in your field

- rapid publication on acceptance

- support for research data, including large and complex data types

- gold Open Access which fosters wider collaboration and increased citations

- maximum visibility for your research: over $100 \mathrm{M}$ website views per year

At $\mathrm{BMC}$, research is always in progress.

Learn more biomedcentral.com/submissions 